\title{
Unsteady Helical Flows of a Size-Dependent Couple-Stress Fluid
}

\author{
Qammar Rubbab, ${ }^{1}$ Itrat Abbas Mirza, ${ }^{2}$ Imran Siddique, ${ }^{3}$ and Saadia Irshad ${ }^{4}$ \\ ${ }^{1}$ Department of Computer Sciences, Air University Multan Campus, Multan, Pakistan \\ ${ }^{2}$ Abdus Salam School of Mathematical Sciences, GC University, Lahore, Pakistan \\ ${ }^{3}$ Department of Mathematics, University of Management and Technology, Sialkot Campus, Pakistan \\ ${ }^{4}$ Department of Management Sciences, Air University Multan Campus, Multan, Pakistan
}

Correspondence should be addressed to Qammar Rubbab; rubabqammar@gmail.com

Received 1 December 2016; Accepted 15 January 2017; Published 8 February 2017

Academic Editor: John D. Clayton

Copyright (C) 2017 Qammar Rubbab et al. This is an open access article distributed under the Creative Commons Attribution License, which permits unrestricted use, distribution, and reproduction in any medium, provided the original work is properly cited.

\begin{abstract}
The helical flows of couple-stress fluids in a straight circular cylinder are studied in the framework of the newly developed, fully determinate linear couple-stress theory. The fluid flow is generated by the helical motion of the cylinder with time-dependent velocity. Also, the couple-stress vector is given on the cylindrical surface and the nonslip condition is considered. Using the integral transform method, analytical solutions to the axial velocity, azimuthal velocity, nonsymmetric force-stress tensor, and couple-stress vector are obtained. The obtained solutions incorporate the characteristic material length scale, which is essential to understand the fluid behavior at microscales. If characteristic length of the couple-stress fluid is zero, the results to the classical fluid are recovered. The influence of the scale parameter on the fluid velocity, axial flow rate, force-stress tensor, and couple-stress vector is analyzed by numerical calculus and graphical illustrations. It is found that the small values of the scale parameter have a significant influence on the flow parameters.
\end{abstract}

\section{Introduction}

The existence of couple stresses in continuum mechanics is a consequence of the discrete character of the matter at the finest scale. Also, the noncentral property of forces between elementary particles of matter leads to the appearing of couple stresses [1]. E. Cosserat and F. Cosserat [2] were the first to have incorporated the couple stresses by considering the oriented material point triads and independent microrotation in theory of continuum solids. Toupin [3], Mindlin and Tiersten [4], and Koiter [5] have developed theories with couple stresses, in which the rigid body motion of the infinitesimal element of matter at each point of the continuum is described by six degrees of freedom.

Based on these theories with couple stresses, Stokes [6] has developed the theory of couple-stress fluids in order to study the size-dependent behavior of flows. Stokes' theory represents the simplest generalization of the classical theory of fluids, which considers the presence of couple stresses. All the above models have various inconsistencies. In the context of Stokes' theory, the indeterminacy of the spherical part of couple-stress tensor is a major shortcoming. Another problem appears in the theory of Mindlin and Tiersten; namely, in their theory, the constitutive relation for the forcestress tensor contains the body couples. The indeterminate spherical part of couple-stress tensor is ignored in Stokes' theory, without justifications. Also, the presence of body couples in the constitutive relations was neglected by Stokes in his theory.

Hadjesfandiari et al. $[1,7,8]$ have solved these issues by using arguments based upon the energy equation along with kinematical considerations. They developed a fully consistent couple-stress theory for solids and fluids, which establishes the couple-stress tensor and mean curvature rate tensor as skew-symmetric energy conjugate measures. Their results could be useful for examining fluid flow problems influenced by the mechanics at small scales. Significant application areas for size-dependent fluid mechanics involve modeling of blood flows, lubrication problems, liquid crystals, and polymeric suspensions. This branch of fluid mechanics has attracted a growing interest from researchers in the field. 
Bakhti and Azrar [9] studied the steady flow of a couplestress fluid through constricted tapered artery under influence of a transverse magnetic field, moving catheter, and slip velocity. Solutions to velocity and shear stress are expressed with Bessel's functions. Pralhad and Schultz [10] used the couple-stress fluid model for the study of the steady flow of blood through stenosed artery. Blood velocity, the resistance to flow, and shear stress distribution have been obtained. Verma et al. [11] have studied the blood flow in a stenosed tube considering the blood as a couple-stress fluid. Effects of slip velocity in stenosed tube were highlighted. Shenoy and Pai [12] have made the static analysis of a misaligned externally adjustable fluid-film bearing including turbulence and couple-stress effects in lubricants blended with polymer additives. Naduvinamani and Patil [13] obtained a numerical solution to finite modified Reynolds equation for couplestress squeeze film lubrication of porous journal bearings.

The unsteady three-dimensional flow of couple-stress fluid over a stretched surface with mass transfer and chemical reaction was investigated by Hayat et al. [14]. Devakar and Iyengar [15] investigated the generalized Stokes' problems for incompressible couple-stress fluids. The effects of kinetic helicity (velocity-vorticity correlation) on turbulent momentum transport were investigated by Yokoi et al. [16, 17]. Other interesting topics can be found in references [18-21].

We must mention that the models considered in the above articles are based on the theory developed by Mindlin, Tiersten, and Stokes.

In the present paper we consider the consistent theory of couple-stress fluids elaborated by Hadjesfandiari and his coworkers $[1,8]$ and, we study the helical flows of couplestress fluids within a straight circular cylinder under general boundary conditions. In the studied problem, the fluid motion is generated by the helical motion of the cylindrical surface with the time-dependent axial and azimuthal velocities and by the time-dependent couple-stress vector on the cylinder surface. The nonslip conditions are, also, considered. Using suitable nondimensional variables, we determine analytical solutions to the axial and azimuthal velocities by means of the integral transform method. The axial angular velocity and the flow rate are also obtained. Components of the nonsymmetric force-stress tensor and the couple-stress vector are determined from the constitutive relations and velocity field. Obviously, if the characteristic length of couple-stress fluids is zero, we recover results for the classical fluid. The obtained analytical solutions are used in order to perform numerical calculations using the Mathcad software for particular external loadings. The results are graphically presented. It is found that all flow parameters are influenced by the fluid scale parameter. The significant influence is obtained for small values of the scale parameter.

\section{Statement of the Problem}

We consider a homogeneous, incompressible, viscous couplestress fluid flowing inside a straight circular cylinder of radius $R$. The cylindrical coordinate system $(r, \theta, z)$ has the $z$-axis identical with the cylinder axis. The governing equations of the couple-stress fluid are as follows $[1,8]$ : (i) Continuity equation:

$$
\nabla \cdot \vec{v}=\frac{\partial v_{r}}{\partial r}+\frac{1}{r} \frac{\partial v_{\theta}}{\partial \theta}+\frac{\partial v_{z}}{\partial z}=0
$$

where $\vec{v}=v_{r} \vec{e}_{r}+v_{\theta} \vec{e}_{\theta}+v_{z} \vec{e}_{z}$ is the fluid velocity.

(ii) Equation of linear momentum (the body forces are neglected):

$$
\rho\left(\frac{\partial \vec{v}}{\partial t}+\nabla \vec{v} \cdot \vec{v}\right)=-\nabla p+\mu \nabla^{2} \vec{v}-\eta \nabla^{2} \nabla^{2} \vec{v},
$$

where $\rho$ is the fluid density, $\mu$ is the dynamic viscosity, $\eta$ is the viscosity coefficient of couple-stress fluid, and $p$ is the thermodynamic pressure.

(iii) The constitutive equations:

The nonsymmetric force-stress tensor:

$$
\mathbf{T}=-p \mathbf{I}+\boldsymbol{\tau}, \quad \boldsymbol{\tau}=2 \mu \mathbf{D}+2 \eta \mathbf{\Omega},
$$

where

$$
\begin{aligned}
& \mathbf{D}=\frac{1}{2}\left[\nabla \vec{v}+(\nabla \vec{v})^{T}\right], \\
& \mathbf{\Omega}=\frac{1}{2}\left[\nabla \vec{v}-(\nabla \vec{v})^{T}\right]
\end{aligned}
$$

represent the strain rate tensor and the angular velocity tensor, respectively.

The polar couple-stress vector:

$$
\vec{M}=2 \eta \Delta \vec{v}
$$

In this paper, we consider that the velocity field and pressure are functions of the form

$$
\begin{aligned}
\vec{v}(r, t) & =v_{\theta}(r, t) \vec{e}_{\theta}+\vec{v}_{z}(r, t) \vec{e}_{z}, \\
p & =p(r, t) .
\end{aligned}
$$

Equation (1) is identically satisfied and (2)-(6) become

$$
\begin{aligned}
\frac{\partial p}{\partial r} & =\frac{\rho}{r} v_{\theta}^{2}, \\
\rho \frac{\partial v_{\theta}}{\partial t} & =\mu\left(\Delta-\frac{1}{r^{2}}\right) v_{\theta}-\eta\left(\Delta-\frac{1}{r^{2}}\right)\left(\Delta-\frac{1}{r^{2}}\right) v_{\theta}, \\
\rho \frac{\partial v_{z}}{\partial t} & =\mu \Delta v_{z}-\eta \Delta^{2} v_{z}, \\
\tau_{r r} & =0, \\
\tau_{r \theta} & =\mu r \frac{\partial}{\partial r}\left(\frac{v_{\theta}}{r}\right)-\eta \Delta\left[\frac{1}{r} \frac{\partial}{\partial r}\left(r v_{\theta}\right)\right], \\
\tau_{r z} & =\mu \frac{\partial v_{z}}{\partial r}-\eta \Delta\left(\frac{\partial v_{z}}{\partial r}\right), \\
\tau_{\theta r} & =\mu r \frac{\partial}{\partial r}\left(\frac{v_{\theta}}{r}\right)+\eta \Delta\left[\frac{1}{r} \frac{\partial}{\partial r}\left(r v_{\theta}\right)\right],
\end{aligned}
$$




$$
\begin{aligned}
\tau_{\theta \theta} & =0 \\
\tau_{\theta z} & =0 \\
\tau_{z r} & =\mu \frac{\partial v_{z}}{\partial r}+\eta \Delta\left(\frac{\partial v_{z}}{\partial r}\right), \\
\tau_{z \theta} & =0 \\
\tau_{z z} & =0 \\
M_{r} & =0 \\
M_{\theta} & =2 \eta\left(\Delta-\frac{1}{r^{2}}\right) v_{\theta}, \\
M_{z} & =2 \eta \Delta v_{z} .
\end{aligned}
$$

We consider the following initial-boundary conditions:

$$
\begin{aligned}
v_{\theta}(r, 0) & =0, \\
v_{z}(r, 0) & =0, \\
v_{\theta}(0, t) & =0, \\
M_{\theta}(0, t) & =0, \\
\left.\frac{\partial v_{z}}{\partial r}\right|_{r=0} & =0, \\
v_{\theta}(R, t) & =V_{1} f_{1}(t), \\
M_{\theta}(R, t) & =2 \eta m_{1} f_{2}(t), \\
v_{z}(R, t) & =V_{0} g_{1}(t), \\
M_{z}(R, t) & =2 \eta m_{2} g_{2}(t) .
\end{aligned}
$$

Functions $f_{1}(t), f_{2}(t), g_{1}(t)$, and $g_{2}(t)$ are piecewise continuous functions on $[0, t]$; for every $t>0$, they have exponential order at infinity and $f_{1}(0)=f_{2}(0)=g_{1}(0)=$ $g_{2}(0)$.

We define the characteristic material length

$$
\ell=\sqrt{\frac{\eta}{\mu}}[\mathrm{m}],
$$

which is absent in classical fluid mechanics but is fundamental for couple-stress fluids.

Introducing the nondimensional variables

$$
\begin{aligned}
& r^{*}=\frac{r}{R}, \\
& t^{*}=\frac{v t}{R^{2}}, \\
& u^{*}=\frac{R v_{\theta}}{v},
\end{aligned}
$$

$$
\begin{aligned}
v^{*} & =\frac{R v_{z}}{v}, \\
p^{*} & =\frac{R^{2} p}{\rho v^{2}}, \\
a & =\frac{\ell^{2}}{R^{2}}, \\
\tau^{*} & =\frac{R^{2}}{\rho v^{2}} \boldsymbol{\tau}, \\
\vec{M}^{*} & =\frac{R}{\rho v^{2}} \vec{M}, \\
f_{1}^{*}\left(t^{*}\right) & =\frac{R V_{1}}{v} f_{1}\left(\frac{R^{2} t^{*}}{v}\right), \\
f_{2}^{*}\left(t^{*}\right) & =\frac{m_{1} R^{3}}{v} f_{2}\left(\frac{R^{2} t^{*}}{v}\right), \\
g_{2}^{*}\left(t^{*}\right) & =\frac{m_{2} R^{3}}{v} g_{2}\left(\frac{R^{2} t^{*}}{v}\right) \\
g_{1}^{*}\left(t^{*}\right) & =\frac{R V_{0}}{v} g_{1}\left(\frac{R^{2} t^{*}}{v}\right),
\end{aligned}
$$

into (8)-(10) and dropping the star notation, we obtain the following nondimensional problem:

$$
\begin{aligned}
\frac{\partial p}{\partial r} & =\frac{1}{r} u^{2} \\
\frac{\partial u}{\partial t} & =\left(\Delta-\frac{1}{r^{2}}\right) u-a\left(\Delta-\frac{1}{r^{2}}\right)\left(\Delta-\frac{1}{r^{2}}\right) u \\
\frac{\partial v}{\partial t} & =\Delta v-a \Delta^{2} v \\
\tau_{r r} & =0 \\
\tau_{r \theta} & =r \frac{\partial}{\partial r}\left(\frac{u}{r}\right)-a \Delta\left[\frac{1}{r} \frac{\partial}{\partial r}(r u)\right] \\
\tau_{r z} & =\frac{\partial v}{\partial r}-a \Delta\left(\frac{\partial v}{\partial r}\right), \\
\tau_{\theta r} & =r \frac{\partial}{\partial r}\left(\frac{u}{r}\right)+a \Delta\left[\frac{1}{r} \frac{\partial}{\partial r}(r u)\right] \\
\tau_{\theta \theta} & =0 \\
\tau_{\theta z} & =0 \\
\tau_{z r} & =\frac{\partial v}{\partial r}+a \Delta\left(\frac{\partial v}{\partial r}\right) \\
\tau_{z \theta} & =0 \\
\tau_{z z} & =0
\end{aligned}
$$




$$
\begin{aligned}
M_{r} & =0, \\
M_{\theta} & =2 a\left(\Delta-\frac{1}{r^{2}}\right) u, \\
M_{z} & =2 a \Delta v, \\
u(r, 0) & =0, \\
v(r, 0) & =0, \\
u(0, t) & =0, \\
M_{\theta}(0, t) & =0, \\
\left.\frac{\partial v}{\partial r}\right|_{r=0} & =0, \\
u(1, t) & =f_{1}(t), \\
M_{\theta}(1, t) & =2 a f_{2}(t), \\
v(1, t) & =g_{1}(t), \\
M_{z}(1, t) & =2 a g_{2}(t) .
\end{aligned}
$$

In the end of this section, we give two lemmas regarding some properties to the operators from (16) and (17). These properties will be used in order to find solutions of the above problem.

Lemma 1. If $\alpha_{n}, n=1,2, \ldots$ are the positive roots of Bessel function $J_{0}(x), v_{n}(t)=\int_{0}^{1} v(r, t) r J_{0}\left(\alpha_{n} r\right) d r$, and $\left.(\partial v(r, t) / \partial r)\right|_{r=0}=0$, then,

(i)

$$
\begin{aligned}
& \int_{0}^{1}(\Delta v(r, t)) r J_{0}\left(\alpha_{n} r\right) d r \\
& \quad=\alpha_{n} J_{1}\left(\alpha_{n}\right) v(1, t)-\alpha_{n}^{2} v_{n}(t)
\end{aligned}
$$

(ii)

$$
\begin{aligned}
\int_{0}^{1}( & \left.\Delta^{2} v(r, t)\right) r J_{0}\left(\alpha_{n} r\right) d r \\
= & -\alpha_{n}^{3} J_{1}\left(\alpha_{n}\right) v(1, t)+\left.\alpha_{n} J_{1}\left(\alpha_{n}\right) \Delta v(r, t)\right|_{r=1} \\
& +\alpha_{n}^{4} v_{n}(t)
\end{aligned}
$$

Lemma 2. If $\beta_{n}, n=1,2, \ldots$ are the positive roots of Bessel function $J_{1}(x), u_{n}(t)=\int_{0}^{1} u(r, t) r J_{1}\left(\beta_{n} r\right) d r$, and $(\Delta-$ $\left.1 / r^{2}\right)\left.u(r, t)\right|_{r=0}=0$, then,

(i)

$$
\begin{gathered}
\int_{0}^{1}\left(\left(\Delta-\frac{1}{r^{2}}\right) u(r, t)\right) r J_{0}\left(\alpha_{n} r\right) d r \\
\quad=-\beta_{n} J_{0}\left(\alpha_{n}\right) u(1, t)-\beta_{n}^{2} u_{n}(t)
\end{gathered}
$$

(ii)

$$
\begin{aligned}
\int_{0}^{1} & \left(\left(\Delta-\frac{1}{r^{2}}\right)^{2} u(r, t)\right) r J_{1}\left(\beta_{n} r\right) d r \\
= & \beta_{n}^{3} J_{0}\left(\beta_{n}\right) u(1, t) \\
& \quad+\left.\beta_{n} J_{0}\left(\beta_{n}\right)\left(\Delta-\frac{1}{r^{2}}\right) u(r, t)\right|_{r=1}+\beta_{n}^{4} u_{n}(t) .
\end{aligned}
$$

The demonstration of relations (26)-(29) is made easy using integration by parts and properties of Bessel functions $[22,23]$. It is noted that $v_{n}(t)$ is zero-order finite Hankel transform of function $v(r, t)$ and $u_{n}(t)$ is the finite Hankel transform of the first order of function $u(r, t)$, respectively.

\section{Solution of the Problem}

In order to find the solution of problem (15)-(25), we use the Laplace transform with respect to the variable time and finite Hankel transform with respect the radial coordinate $[24,25]$.

3.1. Velocity Field. Applying the Laplace and finite Hankel transforms to (16) and (17), using the initial and boundary conditions (22)-(25) and Lemmas 1 and 2, we obtain the following transformed equations:

$$
\begin{aligned}
\bar{u}_{n}(s)= & -\frac{\beta_{n} J_{0}\left(\beta_{n}\right)\left(1+a \beta_{n}^{2}\right)}{s+\beta_{n}^{2}\left(1+a \beta_{n}^{2}\right)} \bar{f}_{1}(s) \\
& +\frac{a \beta_{n} J_{0}\left(\beta_{n}\right)}{s+\beta_{n}^{2}\left(1+a \beta_{n}^{2}\right)} \bar{f}_{2}(s), \\
\bar{v}_{n}(s)= & \frac{\alpha_{n} J_{1}\left(\alpha_{n}\right)\left(1+a \alpha_{n}^{2}\right)}{s+\alpha_{n}^{2}\left(1+a \alpha_{n}^{2}\right)} \bar{g}_{1}(s) \\
& -\frac{a \alpha_{n} J_{1}\left(\alpha_{n}\right)}{s+\alpha_{n}^{2}\left(1+a \alpha_{n}^{2}\right)} \bar{g}_{2}(s),
\end{aligned}
$$

where $\bar{u}_{n}(s)=\int_{0}^{\infty} u_{n}(t) e^{-s t} d t, \bar{v}_{n}(s)=\int_{0}^{\infty} v_{n}(t) e^{-s t} d t, \bar{f}_{1}(s)$, $\bar{f}_{2}(s), \bar{g}_{1}(s)$, and $\bar{g}_{2}(s)$ are the Laplace transforms of functions $u_{n}(t), v_{n}(t), f_{1}(t), f_{2}(t), g_{1}(t)$, and $g_{2}(t)$, respectively. Equations (30) can be written in the suitable forms

$$
\begin{aligned}
\bar{u}_{n}(s)= & -\frac{J_{0}\left(\beta_{n}\right)}{\beta_{n}} \bar{f}_{1}(s)+\frac{J_{0}\left(\beta_{n}\right)}{\beta_{n}} \frac{s \bar{f}_{1}(s)}{s+\beta_{n}^{2}\left(1+a \beta_{n}^{2}\right)} \\
& +\frac{J_{0}\left(\beta_{n}\right)}{\beta_{n}^{3}} \bar{f}_{2}(s)-\frac{J_{0}\left(\beta_{n}\right)}{\beta_{n}^{3}\left(1+a \beta_{n}^{2}\right)} \bar{f}_{2}(s) \\
& -\frac{a J_{0}\left(\beta_{n}\right)}{\beta_{n}\left(1+a \beta_{n}^{2}\right)} \frac{s \bar{f}_{2}(s)}{s+\beta_{n}^{2}\left(1+a \beta_{n}^{2}\right)}, \\
\bar{v}_{n}(s)= & \frac{J_{1}\left(\alpha_{n}\right)}{\alpha_{n}} \bar{g}_{1}(s)-\frac{J_{1}\left(\alpha_{n}\right)}{\alpha_{n}} \frac{\bar{g}_{1}(s)}{s+\alpha \alpha_{n}^{2}\left(1+a \alpha_{n}^{2}\right)} \\
& -\frac{\left(4+\alpha_{n}^{2}\right) J_{1}\left(\alpha_{n}\right)}{4 \alpha_{n}^{3}} \bar{g}_{2}(s)
\end{aligned}
$$




$$
\begin{aligned}
& +\frac{4+\alpha_{n}^{2}\left(1+a \alpha_{n}^{2}\right)}{4 \alpha_{n}^{3}\left(1+a \alpha_{n}^{2}\right)} J_{1}\left(\alpha_{n}\right) \bar{g}_{2}(s) \\
& +\frac{a J_{1}\left(\alpha_{n}\right)}{\alpha_{n}\left(1+a \alpha_{n}^{2}\right)} \frac{s \bar{g}_{2}(s)}{s+\alpha_{n}^{2}\left(1+a \alpha_{n}^{2}\right)} .
\end{aligned}
$$

Now, using the integrals

$$
\begin{gathered}
\int_{0}^{1} r^{2} J_{1}\left(\beta_{n} r\right) d r=-\frac{J_{0}\left(\beta_{n}\right)}{\beta_{n}}, \\
\int_{0}^{1} \frac{r^{3}-r}{8} r J_{1}\left(\beta_{n} r\right) d r=\frac{J_{0}\left(\beta_{n}\right)}{\beta_{n}^{3}}, \\
\int_{0}^{1} r J_{0}\left(\alpha_{n} r\right) d r=\frac{J_{1}\left(\alpha_{n}\right)}{\alpha_{n}}, \\
\int_{0}^{1} \frac{r^{2}-1}{4} r J_{0}\left(\alpha_{n} r\right) d r=-\frac{4+\alpha_{n}^{2}}{4 \alpha_{n}^{3}} J_{1}\left(\alpha_{n}\right),
\end{gathered}
$$

and applying the inverse Hankel and Laplace transforms, we obtain closed forms to azimuthal velocity and axial velocities as

$$
\begin{aligned}
& u(r, t)=r f_{1}(t)+\frac{r^{3}-r}{8} f_{2}(t)-2 f_{2}(t) \\
& \cdot \sum_{n=1}^{\infty} \frac{J_{1}\left(\beta_{n} r\right)}{\beta_{n}^{3}\left(1+a \beta_{n}^{2}\right) J_{0}\left(\beta_{n}\right)}+2 \sum_{n=1}^{\infty} \frac{J_{1}\left(\beta_{n} r\right)}{\beta_{n} J_{0}\left(\beta_{n}\right)} \\
& \cdot \int_{0}^{t} \dot{f}_{1}(\tau) \exp \left[-\beta_{n}^{2}\left(1+a \beta_{n}^{2}\right)(t-\tau)\right] d \tau \\
& -2 a \sum_{n=1}^{\infty} \frac{J_{1}\left(\beta_{n} r\right)}{\beta_{n}\left(1+a \beta_{n}^{2}\right) J_{0}\left(\beta_{n}\right)} \\
& \cdot \int_{0}^{t} \dot{f}_{2}(\tau) \exp \left[-\beta_{n}^{2}\left(1+a \beta_{n}^{2}\right)(t-\tau)\right] d \tau \text {, } \\
& v(r, t)=g_{1}(t)+\frac{r^{2}-1}{4} g_{2}(t)+2 g_{2}(t) \\
& \cdot \sum_{n=1}^{\infty} \frac{4+\alpha_{n}^{2}\left(1+a \alpha_{n}^{2}\right) J_{0}\left(\alpha_{n} r\right)}{4 \alpha_{n}^{3}\left(1+a \alpha_{n}^{2}\right) J_{1}\left(\alpha_{n}\right)}-2 \sum_{n=1}^{\infty} \frac{J_{0}\left(\alpha_{n} r\right)}{\alpha_{n} J_{1}\left(\alpha_{n}\right)} \\
& \cdot \int_{0}^{t} \dot{g}_{1}(\tau) \exp \left[-\alpha_{n}^{2}\left(1+a \alpha_{n}^{2}\right)(t-\tau)\right] d \tau \\
& +2 a \sum_{n=1}^{\infty} \frac{J_{0}\left(\alpha_{n} r\right)}{\alpha_{n}\left(1+a \alpha_{n}^{2}\right) J_{1}\left(\alpha_{n}\right)} \\
& \cdot \int_{0}^{t} \dot{g}_{2}(\tau) \exp \left[-\alpha_{n}^{2}\left(1+a \alpha_{n}^{2}\right)(t-\tau)\right] d \tau .
\end{aligned}
$$

In the above relations we have used the notation $\dot{f}(t)=$ $d f(t) / d t$.

By introducing notations

$$
\begin{gathered}
A_{n}(t)=\frac{-2 f_{2}(t)}{\beta_{n}^{3}\left(1+a \beta_{n}^{2}\right) J_{0}\left(\beta_{n}\right)}+\frac{2}{\beta_{n} J_{0}\left(\beta_{n}\right)} \\
\cdot \int_{0}^{t} \dot{f}_{1}(\tau) \exp \left[-\beta_{n}^{2}\left(1+a \beta_{n}^{2}\right)(t-\tau)\right] d \tau
\end{gathered}
$$

$$
\begin{gathered}
-\frac{2 a}{\beta_{n}\left(1+a \beta_{n}^{2}\right) J_{0}\left(\beta_{n}\right)} \\
\cdot \int_{0}^{t} \dot{f}_{2}(\tau) \exp \left[-\beta_{n}^{2}\left(1+a \beta_{n}^{2}\right)(t-\tau)\right] d \tau \\
B_{n}(t)=\frac{2 g_{2}(t)\left(4+\alpha_{n}^{2}\left(1+a \alpha_{n}^{2}\right)\right)}{4 \alpha_{n}^{3}\left(1+a \alpha_{n}^{2}\right) J_{1}\left(\alpha_{n}\right)}-\frac{2}{\alpha_{n} J_{1}\left(\alpha_{n}\right)} \\
\cdot \int_{0}^{t} \dot{g}_{1}(\tau) \exp \left[-\alpha_{n}^{2}\left(1+a \alpha_{n}^{2}\right)(t-\tau)\right] d \tau \\
+\frac{2 a}{\alpha_{n}\left(1+a \alpha_{n}^{2}\right) J_{1}\left(\alpha_{n}\right)} \\
\cdot \int_{0}^{t} \dot{g}_{2}(\tau) \exp \left[-\alpha_{n}^{2}\left(1+a \alpha_{n}^{2}\right)(t-\tau)\right] d \tau
\end{gathered}
$$

(33) are written as

$$
\begin{aligned}
& u(r, t)=r f_{1}(t)+\frac{r^{3}-r}{8} f_{2}(t)+\sum_{n=1}^{\infty} A_{n}(t) J_{1}\left(\beta_{n} r\right), \\
& v(r, t)=g_{1}(t)+\frac{r^{2}-1}{4} g_{2}(t)+\sum_{n=1}^{\infty} B_{n}(t) J_{0}\left(\alpha_{n} r\right) .
\end{aligned}
$$

It is observed that functions $A_{n}(t)$ and $B_{n}(t)$ have the property $A_{n}(0)=B_{n}(0)=0$.

The axial angular velocity (the spin vector) is given by

$$
\begin{aligned}
\vec{\omega}= & \frac{1}{2} \nabla \times \vec{v}=-\frac{1}{2} \frac{\partial v}{\partial r} \vec{e}_{\theta}+\left(\frac{\partial u}{\partial r}+\frac{u}{r}\right) \vec{e}_{z} \\
= & \frac{1}{2}\left(\sum_{n=1}^{\infty} \alpha_{n} B_{n}(t) J_{1}\left(\alpha_{n} r\right)-\frac{r}{2} g_{2}(t)\right) \vec{e}_{\theta} \\
& +\frac{1}{2}\left(2 f_{1}(t)+\frac{2 r^{2}-1}{4} f_{2}(t)\right. \\
& \left.+\sum_{n=1}^{\infty} \beta_{n} A_{n}(t) J_{0}\left(\beta_{n} r\right)\right) \vec{e}_{z},
\end{aligned}
$$

and the flow rate is

$$
\begin{aligned}
Q(t) & =\int_{0}^{1} 2 \pi r v(r, t) d r \\
& =\pi g_{1}(t)-\frac{\pi}{8} g_{2}(t)+2 \pi \sum_{n=1}^{\infty} \frac{B_{n}(t)}{\alpha_{n}} J_{1}\left(\alpha_{n}\right) .
\end{aligned}
$$

3.2. Force-Stress Tensor and Couple-Stress Vector. Replacing (35) in (18)-(21) and performing calculations, we get the following expressions for the force-stresses and couple-stress vector:

$$
\begin{aligned}
\tau_{r \theta}= & \left(\frac{r^{2}}{4}-2 a\right) f_{2}(t) \\
& +\sum_{n=1}^{\infty} \beta_{n} A_{n}(t)\left(1+2 a \beta_{n}^{2}\right) J_{0}\left(\beta_{n} r\right) \\
& -\frac{2}{r} \sum_{n=1}^{\infty} A_{n}(t) J_{1}\left(\beta_{n} r\right)
\end{aligned}
$$




$$
\begin{aligned}
\tau_{\theta r}= & \left(\frac{r^{2}}{4}+2 a\right) f_{2}(t) \\
& +\sum_{n=1}^{\infty} \beta_{n} A_{n}(t)\left(1-2 a \beta_{n}^{2}\right) J_{0}\left(\beta_{n} r\right) \\
& -\frac{2}{r} \sum_{n=1}^{\infty} A_{n}(t) J_{1}\left(\beta_{n} r\right), \\
\tau_{r z}= & \frac{r^{2}-a}{2 r} g_{2}(t) \\
& -\sum_{n=1}^{\infty} \alpha_{n} B_{n}(t)\left[1+a\left(\alpha_{n}^{2}-\frac{1}{r^{2}}\right)\right] J_{1}\left(\alpha_{n} r\right), \\
\tau_{z r}= & \frac{r^{2}+a}{2 r} g_{2}(t) \\
& -\sum_{n=1}^{\infty} \alpha_{n} B_{n}(t)\left[1-a\left(\alpha_{n}^{2}-\frac{1}{r^{2}}\right)\right] J_{1}\left(\alpha_{n} r\right), \\
M_{z}= & \tau_{\theta \theta}=\tau_{\theta z}=\tau_{z \theta}=\tau_{z z}=0, \\
M_{r}= & 0, \\
M_{\theta} & 2 a\left[r f_{2}(t)-\sum_{n=1}^{\infty} \beta_{n}^{2} A_{n}(t) J_{1}\left(\beta_{n} r\right)\right], \\
& {\left[\alpha_{n=1}^{2} B_{n}(t) J_{0}\left(\alpha_{n} r\right)\right] . }
\end{aligned}
$$

It is easy to see that, for $a=0$ (the ordinary Newtonian fluid), the force-stress tensor becomes a symmetric tensor and the couple-stress vector is zero.

3.3. Particular Case (Constant Velocity and Couple Stress on the Boundary). Let us consider the following boundary conditions:

$$
\begin{aligned}
& f_{1}(t)=p_{1} H(t), \\
& f_{2}(t)=p_{2} H(t), \\
& g_{1}(t)=q_{1} H(t), \\
& g_{2}(t)=q_{2} H(t),
\end{aligned}
$$

where $p_{1} \geq 0, p_{2} \geq 0, q_{1} \geq 0$, and $q_{2} \geq 0$ are constants and $H(t)=(1 / 2) \operatorname{sign}(t)(1+\operatorname{sign}(t))$ is the Heaviside unit step function. In this case, the derivatives of functions given by (39) are

$$
\begin{aligned}
& \dot{f}_{1}(t)=p_{1} \delta(t), \\
& \dot{f}_{2}(t)=p_{2} \delta(t), \\
& \dot{g}_{1}(t)=q_{1} \delta(t), \\
& \dot{g}_{2}(t)=q_{2} \delta(t),
\end{aligned}
$$

$\delta(t)$ being the Dirac distribution. Functions $A_{n}(t)$ and $B_{n}(t)$ are written in simpler forms, as

$$
\begin{aligned}
& A_{n}(t)=\frac{-2 p_{2} H(t)}{\beta_{n}^{3}\left(1+a \beta_{n}^{2}\right) J_{0}\left(\beta_{n}\right)} \\
& +\frac{2 H(t)}{\beta_{n} J_{0}\left(\beta_{n}\right)}\left[p_{1}-\frac{a p_{2}}{1+a \beta_{n}^{2}}\right] \\
& \quad \cdot \exp \left[-\beta_{n}^{2}\left(1+a \beta_{n}^{2}\right) t\right] \\
& B_{n}(t)=\frac{2 q_{2} H(t)\left(4+\alpha_{n}^{2}\left(1+a \alpha_{n}^{2}\right)\right)}{4 \alpha_{n}^{3}\left(1+a \alpha_{n}^{2}\right) J_{1}\left(\alpha_{n}\right)} \\
& -\frac{2 H(t)}{\alpha_{n} J_{1}\left(\alpha_{n}\right)}\left[q_{1}-\frac{a q_{2}}{1+a \alpha_{n}^{2}}\right] \exp \left[-\alpha_{n}^{2}\left(1+a \alpha_{n}^{2}\right) t\right] .
\end{aligned}
$$

\section{Numerical Results and Discussion}

Unsteady helical flows in the consistent theory of couplestress fluids were considered, under general boundary conditions. By using suitable nondimensional variables, the governing flow equations are obtained in the dimensionless form. It is important to note that these equations contain as parameter the dimensionless scale flow parameter $a$, defined as the square of the rate between the characteristic material length and the radius of circular cylinder. As a result from the studied particular problems, the scale parameter has a significant influence on the fluid behavior. Obviously, if the scale parameter equals zero, results corresponding to the Newtonian fluid are obtained. Solutions for fluid velocity, nonsymmetric force-stress tensor, and couple-stress vector were obtained using integral transforms method (Laplace transform with respect to the time variable and finite Hankel transform with respect to the radial coordinate). The axial angular velocity and the flow rate are also determined.

The obtained solutions contain in their expressions the positive roots of the Bessel functions $J_{0}(x)$ and $J_{1}(x)$, denoted by $\alpha_{n}$ and $\beta_{n}$, roots which were generated by means of Mathcad subroutine " $\operatorname{root}(f(x), x, a, b)$." In the numerical simulations, we have used $n \in[500,1000]$, values for which the numerical approximation accuracy is very good.

In our study, the azimuthal velocity, axial velocity, and the components of the couple-stress vector are given on the cylinder surface as arbitrary functions of the time $t$; therefore, the obtained solutions can generate solutions to various problems with practical applications.

The numerical results for Figures $1-3$ and for Table 1 were generated under conditions $f_{1}(t)=f_{2}(t)=g_{1}(t)=g_{2}(t)=$ $H(t)=(1 / 2) \operatorname{sign}(t)(1+\operatorname{sign}(t))$.

Figures 4 and 5 were plotted under conditions

$$
\begin{aligned}
& f_{1}(t)=g_{1}(t)=0.2 H(t)=0.1 \operatorname{sign}(t)(1+\operatorname{sign}(t)), \\
& f_{2}(t)=0 \\
& g_{2}(t)=0 .
\end{aligned}
$$



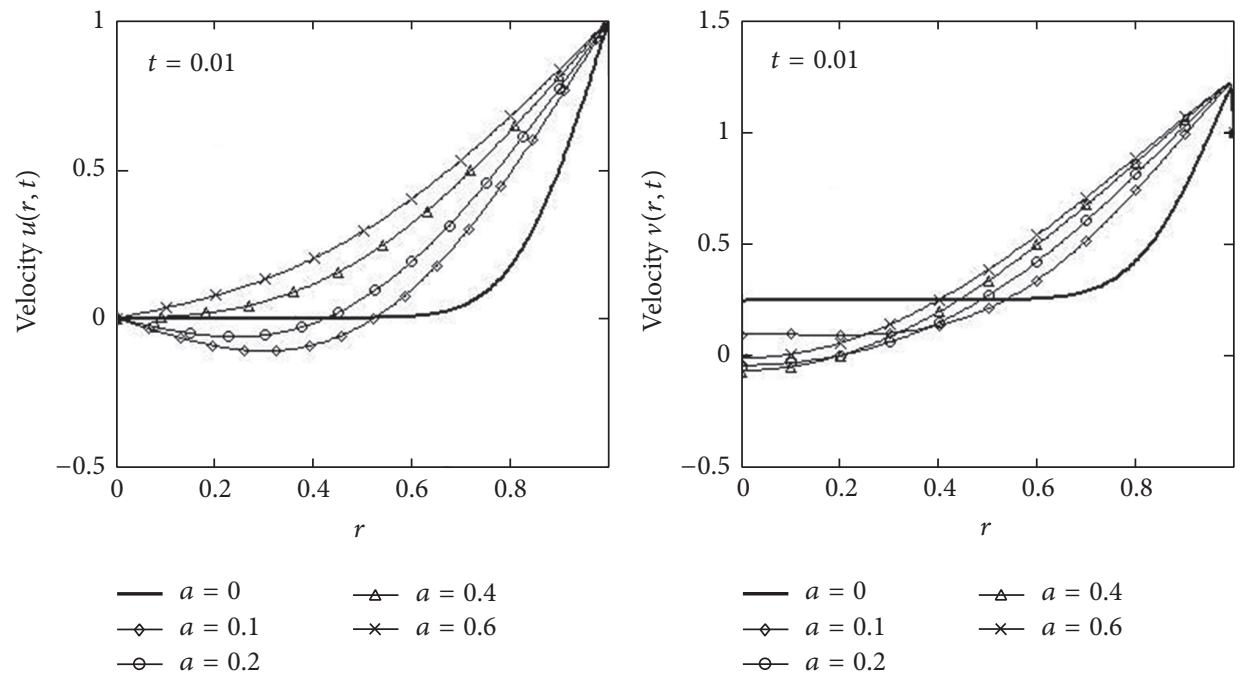

(a)
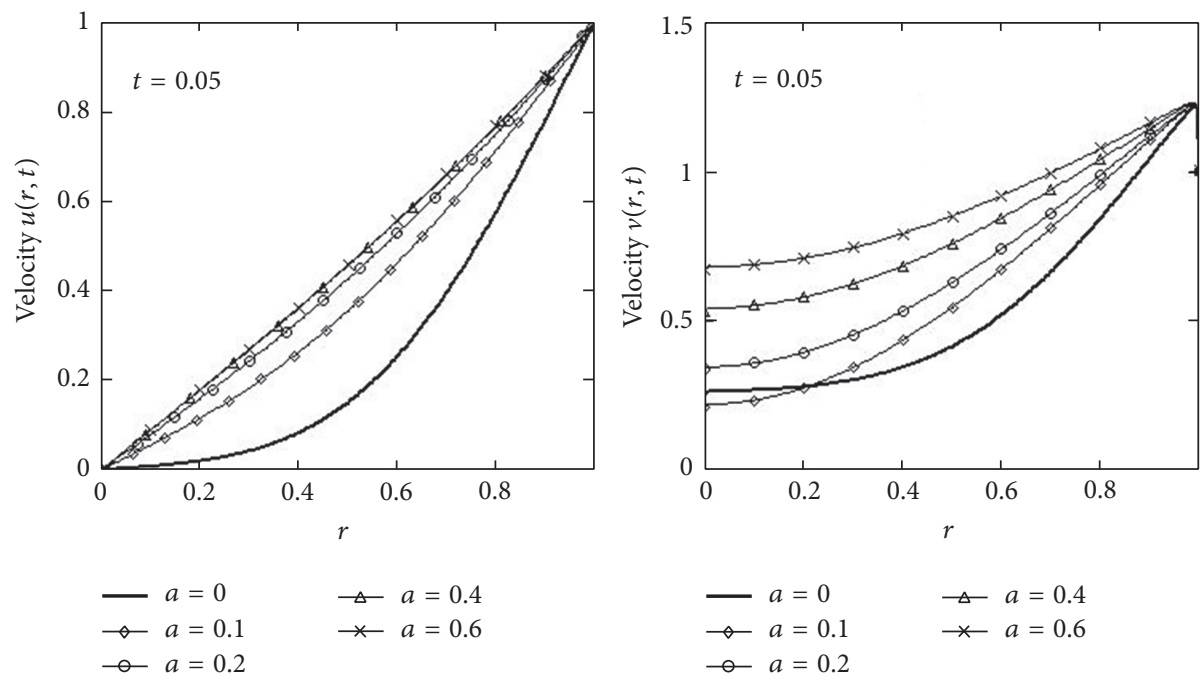

(b)
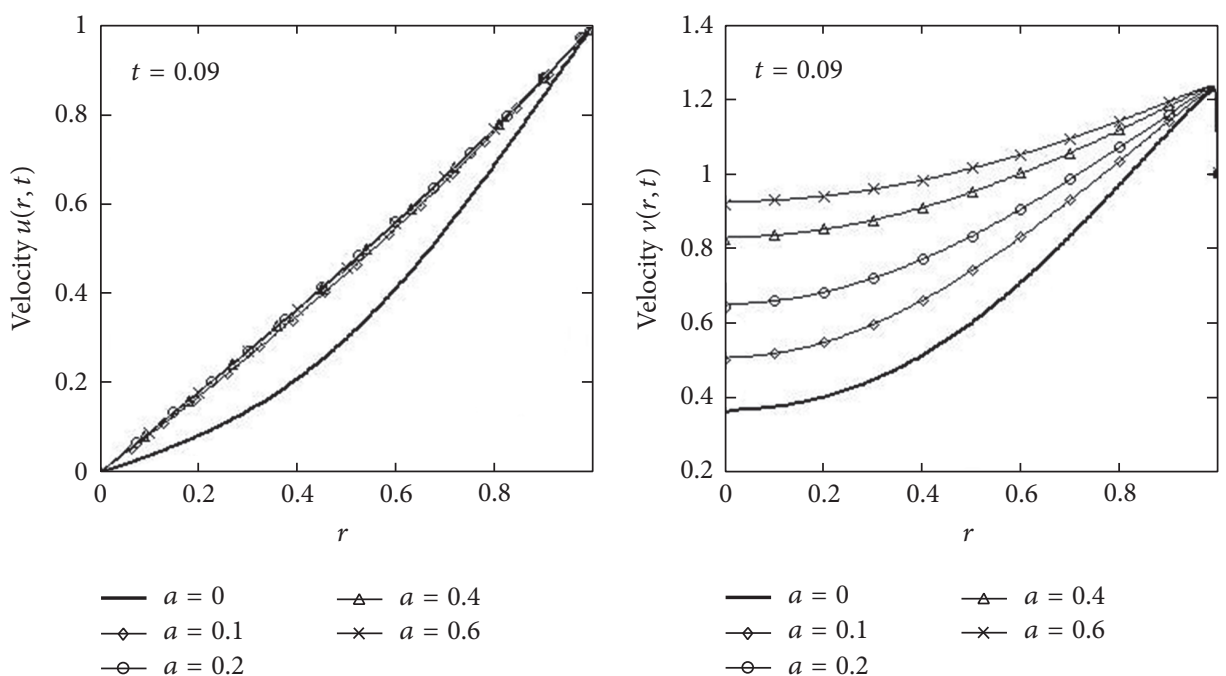

(c)

FIGURE 1: Profiles of azimuthal velocity $u(r, t)$ and axial velocity $v(r, t)$ for small time and different values of scale parameter $a$. 

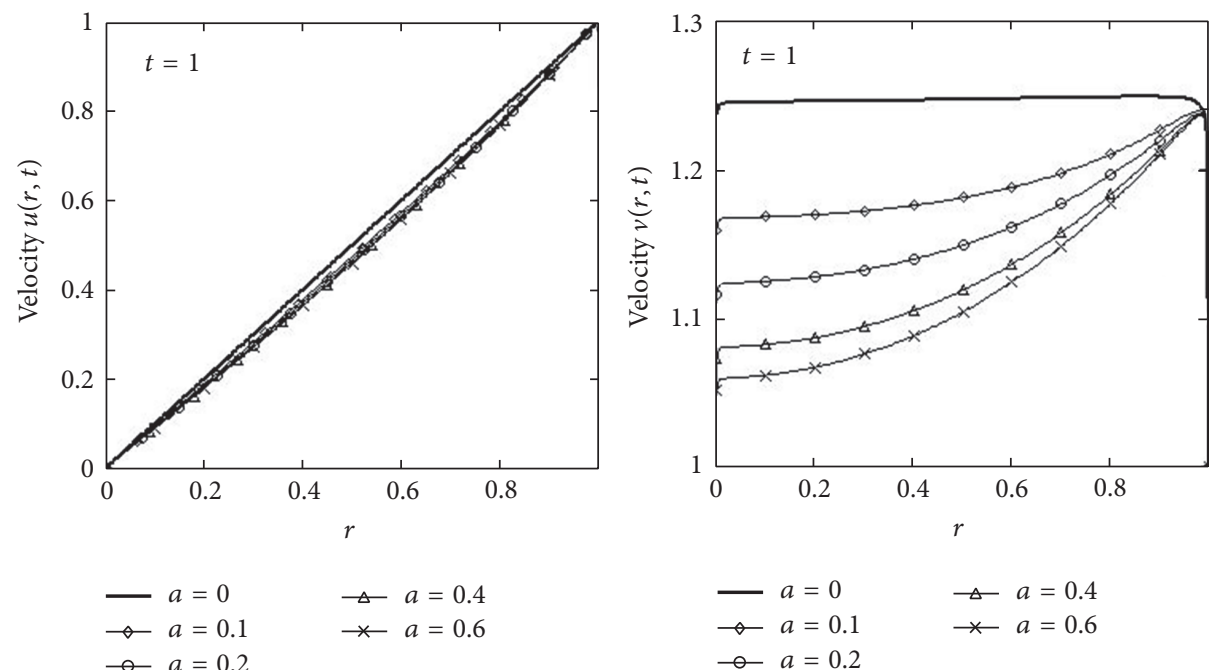

FIgURE 2: Profiles of azimuthal velocity $u(r, t)$ and axial velocity $v(r, t)$ for $t=1$ and different values of the scale parameter $a$.

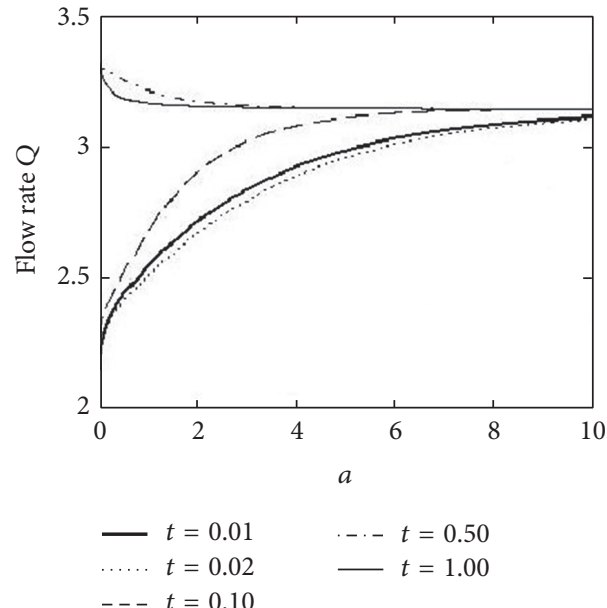

(a)
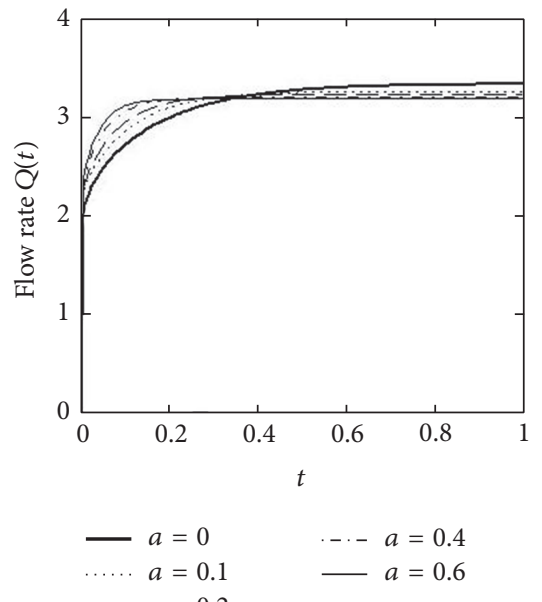

(b)

FIGURE 3: Variation of flow rate with the scale parameter $a$, respectively, with the time $t$.
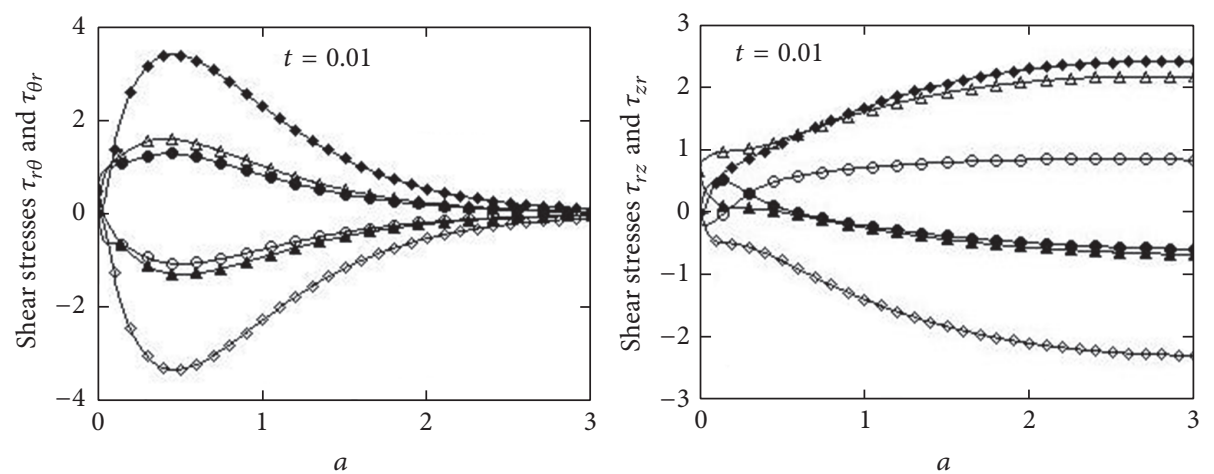

$$
\begin{aligned}
& \stackrel{\tau_{\theta r}}{\longrightarrow} \quad r=0.25 \\
& \tau_{r \theta} \\
& \rightarrow r=0.50 \\
& \rightarrow r=0.25 \\
& ₫ r=0.85 \\
& \therefore r=0.85
\end{aligned}
$$

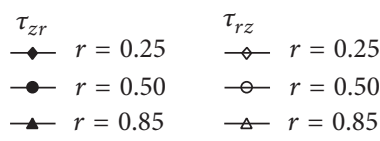

FIGURE 4: The variation of shear stresses with the scale parameter $a$. 

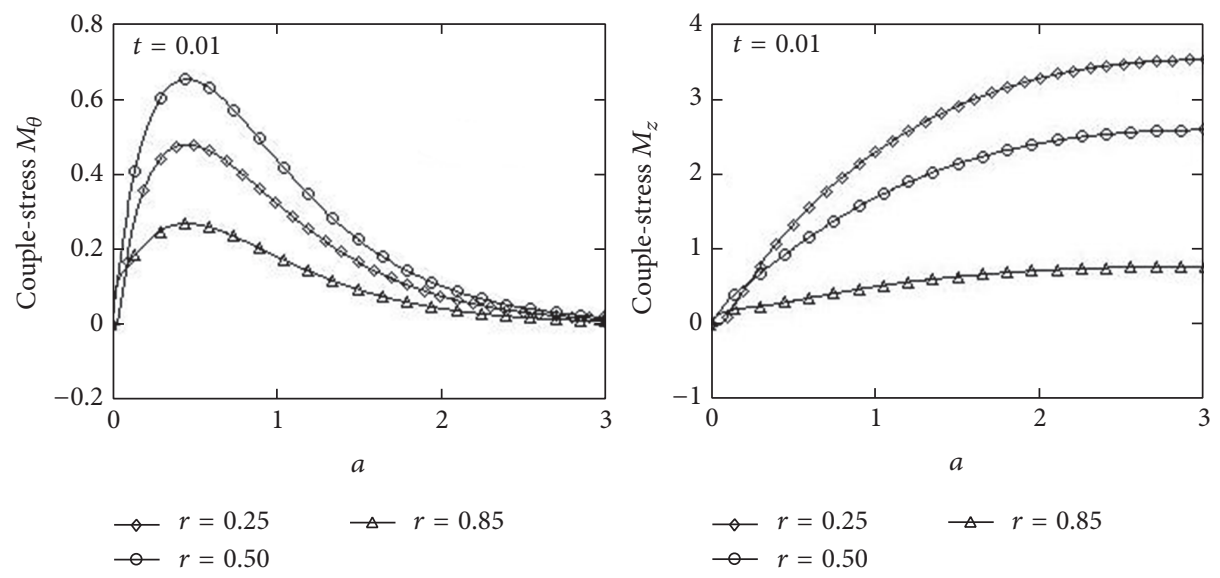

Figure 5: Variation of the couple stress with the scale parameter $a$.

TABLE 1: The influence of time values on fluid velocity components.

(a)

\begin{tabular}{lccc}
\hline & \multicolumn{3}{c}{$a=0.1$} \\
$r$ & $u(r, t), t=1$ & $u(r, t), t=5$ & $u(r, t), t=10$ \\
\hline 0 & 0 & 0 & 0 \\
0.1 & 0.093499 & 0.093499 & 0.093499 \\
0.2 & 0.187262 & 0.187262 & 0.187262 \\
0.3 & 0.281577 & 0.281577 & 0.281577 \\
0.4 & 0.376776 & 0.376776 & 0.376776 \\
0.5 & 0.473268 & 0.473268 & 0.473268 \\
0.6 & 0.57157 & 0.57157 & 0.57157 \\
0.7 & 0.672355 & 0.672355 & 0.672355 \\
0.8 & 0.776506 & 0.776506 & 0.776506 \\
0.9 & 0.885198 & 0.885198 & 0.885198 \\
1 & 1 & 1 & 1 \\
\hline
\end{tabular}

(b)

\begin{tabular}{lccc}
\hline & \multicolumn{3}{c}{$a=0.1$} \\
$r$ & $v(r, t), t=1$ & $v(r, t), t=5$ & $v(r, t), t=10$ \\
\hline 0 & 1.158949 & 1.159123 & 1.159112 \\
0.1 & 1.167755 & 1.167927 & 1.167916 \\
0.2 & 1.169256 & 1.169421 & 1.16941 \\
0.3 & 1.171729 & 1.171882 & 1.171872 \\
0.4 & 1.175404 & 1.17554 & 1.175532 \\
0.5 & 1.180568 & 1.180685 & 1.180677 \\
0.6 & 1.187616 & 1.187711 & 1.187705 \\
0.7 & 1.197082 & 1.197153 & 1.197149 \\
0.8 & 1.209657 & 1.209704 & 1.209701 \\
0.9 & 1.226044 & 1.226067 & 1.226065 \\
1 & 1 & 1 & 1 \\
\hline
\end{tabular}

Figure 1 shows profiles of both azimuthal and axial velocities, versus radial coordinate $r$ for different values of the scale parameter $a$ and for three small values of the time $t$.

The influence of the scale parameter $a$ on rotational velocity is significant only for very small values of the time $t$. It is observed from Figure 1(a) that azimuthal velocity increases with the scale parameter $a$. The rotational velocity of couplestress fluid is bigger than the velocity of classical fluid, except the case of very small values of time $t$. In this particular case, there are values of the scale parameter, for which the couplestress fluid flows more slowly than the Newtonian fluid in the central area of the flow domain. The influence of the scale parameter on the axial velocity is more significant than on the azimuthal velocity. It is seen from Figure 1(b) that the axial velocity increases with the scale parameter.

As is apparent from Figure 2 and values of Table 1, the fluid azimutal velocity is almost steady and the axial velocity has a small time-variation, for $t>1$. These properties are due to the exponential terms in (34) which tend fast to zero, because,

$$
\begin{aligned}
& \alpha_{n}^{2}\left(1+a \alpha_{n}^{2}\right) \geq 5.783(1+5.783 a) \\
& n \geq 1, \alpha_{1} \simeq 2.405, \\
& \beta_{n}^{2}\left(1+a \beta_{n}^{2}\right) \geq 14.682(1+14.682 a), \\
& n \geq 1, \quad \beta_{1} \simeq 3.832
\end{aligned}
$$

(For $t>1$ and $a \geq 0$ the exponential terms in (34) are negligible).

The influence of the scale parameter $a$ on the flow rate $Q(t)$ in axial direction is presented in Figure 3. Obviously, the significant variation of the flow rate is for small values of the time $t$. It is important to note that, for large values of the scale parameter $a$ or for large values of the time $t$, the flow rate in the axial direction becomes constant.

The influence of the scale parameter $a$ on the components $\tau_{r \theta}, \tau_{\theta r}, \tau_{r z}$, and $\tau_{z r}$ of the force-stress tensor and on the components $M_{\theta}$ and $M_{z}$ of the couple-stress vector is analyzed in Figures 4 and 5.

The significant influence occurs for small values of the scale parameter. For these values the shear stresses $\tau_{r \theta}$ and $\tau_{\theta r}$ and the couple-stress component $M_{\theta}$ have an extreme value and tend to approach zero for large values of the scale parameter. 
Shear stresses $\tau_{r z}$ and $\tau_{z r}$ and the couple-stress component $M_{z}$ are monotone increasing/decreasing for small values of the parameter $a$ and tend to a constant value if the parameter values are large.

\section{Competing Interests}

It is hereby declared that none of the authors have any competing interests in the manuscript.

\section{References}

[1] A. R. Hadjesfandiari, G. F. Dargush, and A. Hajesfandiari, "Consistent skew-symmetric couple stress theory for size-dependent creeping flow," Journal of Non-Newtonian Fluid Mechanics, vol. 196, pp. 83-94, 2013.

[2] E. Cosserat and F. Cosserat, Theorie des Corps Deformables, A. Hermann et fils, Paris, France, 1909.

[3] R. A. Toupin, "Elastic materials with couple-stresses," Archive for Rational Mechanics and Analysis, vol. 11, pp. 385-414, 1962.

[4] R. D. Mindlin and H. F. Tiersten, "Effects of couple-stresses in linear elasticity," Archive for Rational Mechanics and Analysis, vol. 11, pp. 415-448, 1962.

[5] W. T. Koiter, "Couple-stresses in the theory of elasticity I and II," Proceedings of the Koninklijke Nederlandse Akademie van Wetenschappen, Series B, Physical Sciences, vol. 67, pp. 17-44, 1967.

[6] V. K. Stokes, "Couple stresses in fluids," Physics of Fluids, vol. 9, no. 9, pp. 1709-1715, 1966.

[7] A. R. Hadjesfandiari and G. F. Dargush, "Couple stress theory for solids," International Journal of Solids and Structures, vol. 48, no. 18, pp. 2496-2510, 2011.

[8] A. R. Hadjesfandiari, A. Hajesfandiari, and G. F. Dargush, "Skew-symmetric couple-stress fluid mechanics," Acta Mechanica, vol. 226, no. 3, pp. 871-895, 2015.

[9] H. Bakhti and L. Azrar, "Steady flow of couple-stress fluid in constricted tapered artery: effects of transverse magnetic field, moving catheter, and slip velocity," Journal of Applied Mathematics, vol. 2016, Article ID 9289684, 11 pages, 2016.

[10] R. N. Pralhad and D. H. Schultz, "Modeling of arterial stenosis and its applications to blood diseases," Mathematical Biosciences, vol. 190, no. 2, pp. 203-220, 2004.

[11] V. K. Verma, M. P. Singh, and V. K. Katiyar, "Mathematical modeling of blood flow through stenosed tube," Journal of Mechanics in Medicine and Biology, vol. 8, no. 1, pp. 27-32, 2008.

[12] B. S. Shenoy and R. Pai, "Effect of turbulence on the static performance of a misaligned externally adjustable fluid film bearing lubricated with couple stress fluids," Tribology International, vol. 44, no. 12, pp. 1774-1781, 2011.

[13] N. B. Naduvinamani and S. B. Patil, "Numerical solution of finite modified Reynolds equation for couple stress squeeze film lubrication of porous journal bearings," Computers and Structures, vol. 87, no. 21-22, pp. 1287-1295, 2009.

[14] T. Hayat, M. Awais, A. Safdar, and A. A. Hendi, "Unsteady three dimensional flow of couple stress fluid over a stretching surface with chemical reaction," Nonlinear Analysis: Modelling and Control, vol. 17, no. 1, pp. 47-59, 2012.

[15] M. Devakar and T. K. V. Iyengar, "Generalized Stokes' problems for an incompressible couple stress fluid," International Journal of Mathematical, Computational, Physical, Electrical and Computer Engineering, vol. 8, no. 1, pp. 113-116, 2014.
[16] N. Yokoi and A. Yoshizawa, "Statistical analysis of the effects of helicity in inhomogeneous turbulence," Physics of Fluids A, vol. 5, no. 2, pp. 464-477, 1993.

[17] N. Yokoi and A. Brandenburg, "Large-scale flow generation by inhomogeneous helicity," Physical Review E-Statistical, Nonlinear, and Soft Matter Physics, vol. 93, no. 3, Article ID 033125, 2016.

[18] F. Awad, N. A. H. Haroun, P. Sibanda, and M. Khumalo, "On couple stress effects on unsteady nanofluid flow over stretching surfaces with vanishing nanoparticle flux at the wall," Journal of Applied Fluid Mechanics, vol. 9, no. 4, pp. 1937-1944, 2016.

[19] S. Ahmed, O. Anwar Bég, and S. K. Ghosh, "A couple stress fluid modeling on free convection oscillatory hydromagnetic flow in an inclined rotating channel," Ain Shams Engineering Journal, vol. 5, no. 4, pp. 1249-1265, 2014.

[20] Y. Kwon, "Incompressible limit for the compressible flows of nematic liquid crystals in the whole space," Advances in Mathematical Physics, vol. 2015, Article ID 427865, 7 pages, 2015.

[21] B. Panicaud and E. Rouhaud, "Derivation of Cosserat's medium equations using different multi-dimensional frameworks," Acta Mechanica, vol. 227, no. 2, pp. 367-385, 2016.

[22] Y. A. Brychkov, Handbook of Special Functions. Derivatives, Integrals, Series and Other Formulas, Chapman and Hall/CRC, Boca Raton, Fla, USA, 2008.

[23] W. Magnus, F. Oberhettinger, and R. P. Soni, Formulas and Theorems for the Special Functions of Mathematical Physics, Springer, New York, NY, USA, 1966.

[24] L. Debnath and D. Bhatta, Integral Transforms and Their Applications, Chapman \& Hall/CRC, Boca Raton, Fla, USA, 2nd edition, 2007.

[25] J. Kang, Y. Liu, and T. Xia, "Unsteady flows of a generalized fractional Burgers' fluid between two side walls perpendicular to a plate," Advances in Mathematical Physics, vol. 2015, Article ID 521069, 9 pages, 2015. 


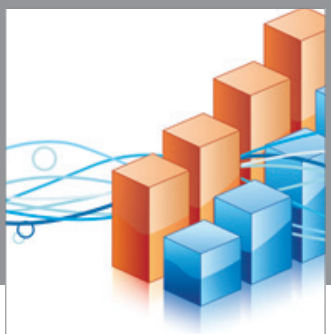

Advances in

Operations Research

vatem alat4

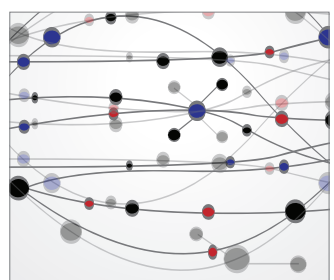

\section{The Scientific} World Journal
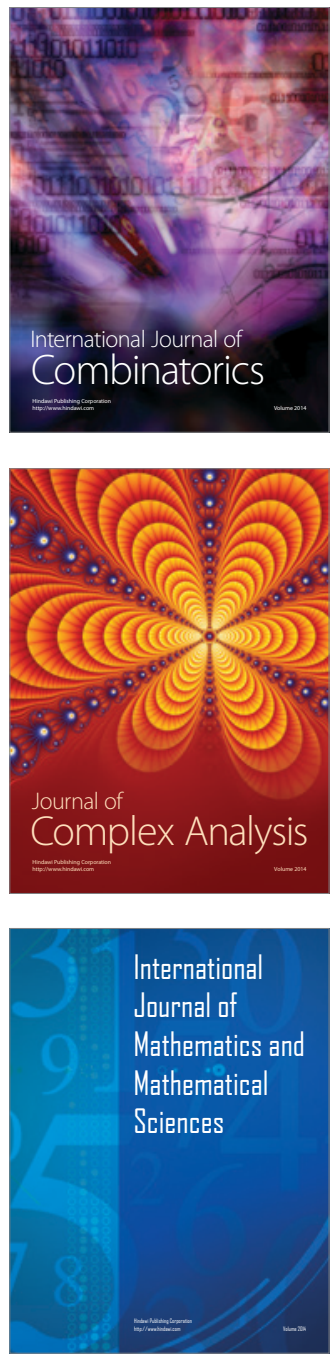
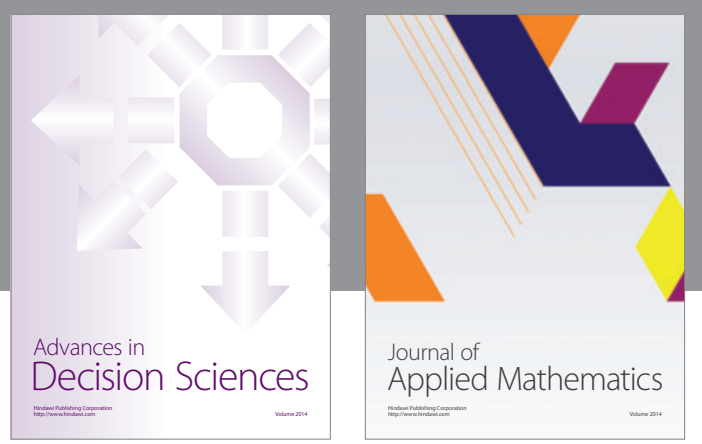

Algebra

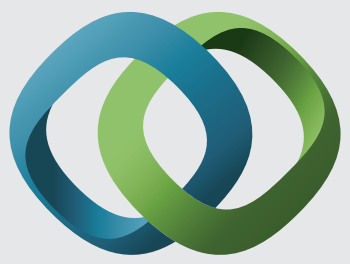

\section{Hindawi}

Submit your manuscripts at

https://www.hindawi.com
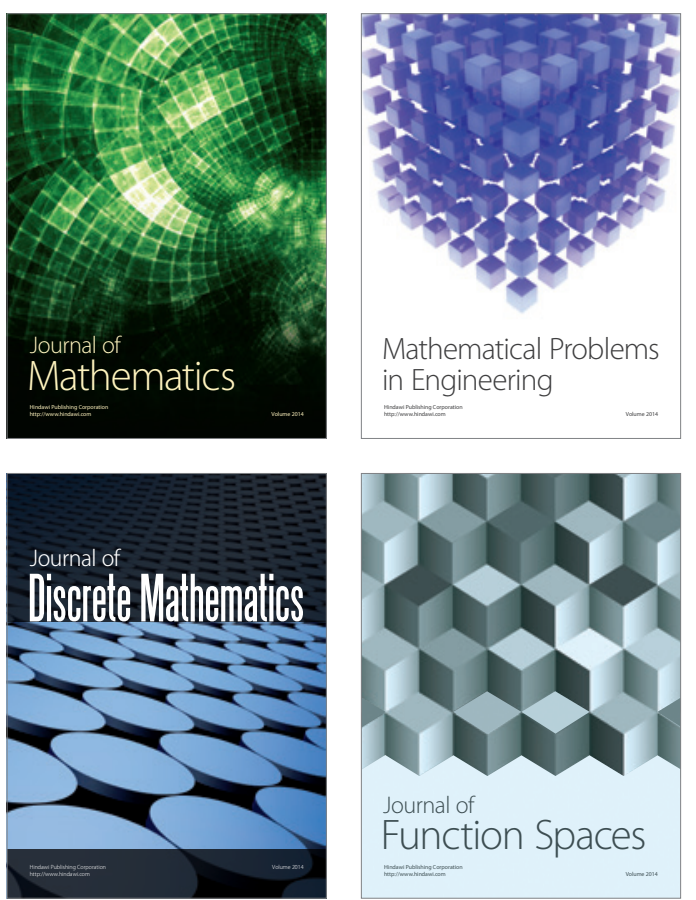

Mathematical Problems in Engineering
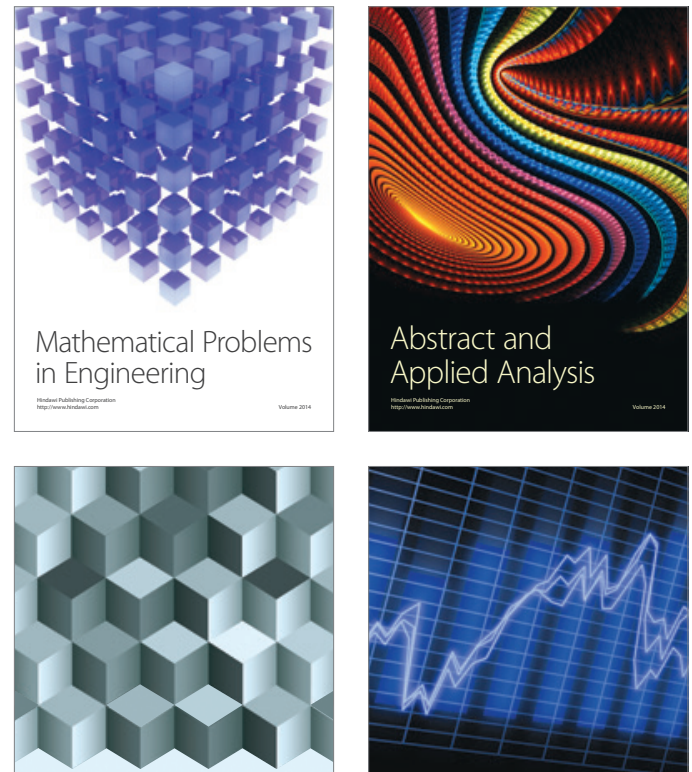

Journal of

Function Spaces

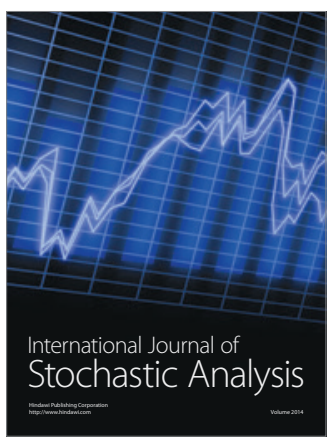

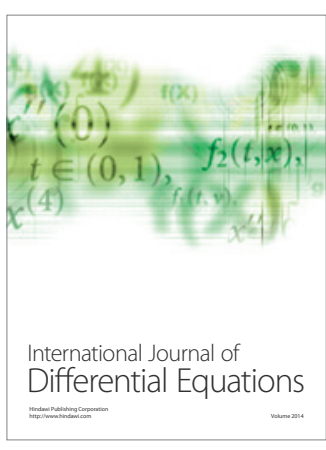
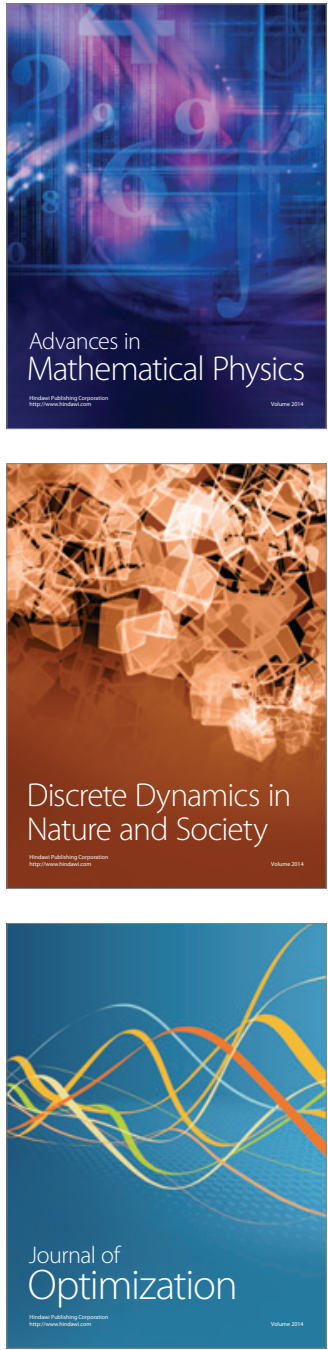